\title{
Extended Plasticity of Visual Cortex in Dark-Reared Animals May Result from Prolonged Expression of cpg15-Like Genes
}

\author{
Wei-Chung Allen Lee and Elly Nedivi \\ Center for Learning and Memory, Departments of Brain and Cognitive Sciences and Biology, Massachusetts Institute of \\ Technology, Cambridge, Massachusetts 02139
}

\begin{abstract}
cpg15 is an activity-regulated gene that encodes a membranebound ligand that coordinately regulates growth of apposing dendritic and axonal arbors and the maturation of their synapses. These properties make it an attractive candidate for participating in plasticity of the mammalian visual system. Here we compare cpg15 expression during normal development of the rat visual system with that seen in response to dark rearing, monocular blockade of retinal action potentials, or monocular deprivation. Our results show that the onset of cpg15 expression in the visual cortex is coincident with eye opening, and it increases until the peak of the critical period at postnatal day 28 (P28). This early expression is independent of both retinal activity and visual experience. After P28, a component of cpg15 expression in the visual cortex, lateral geniculate nucleus (LGN), and superior colliculus (SC) develops a progressively
\end{abstract}

The critical period for susceptibility to environmental influences is one of the central concepts to emerge from studies of visual system development. The capacity for change in response to manipulation of patterned visual input is normally restricted to a defined age window during postnatal development (Hubel and Wiesel, 1970; Hubel et al., 1977). However, in kittens or rats dark-reared (DR) past the end of the normal critical period, manipulation of visual input results in significant plasticity of cortical binocular connectivity as measured by electrophysiological recordings (Cynader and Mitchell, 1980; Mower et al., 1981; Fagiolini et al., 1994; Guire et al., 1999). This prolonged plasticity is not accompanied by a prolonged plasticity measured by anatomical methods. When visualized by autoradiography of geniculocortical afferents, the segregation of ocular dominance columns is incomplete in DR cats and is not altered by later visual manipulations (Mower et al., 1985). The complexity of interpreting dark-rearing experiments highlights our poor understanding of the molecular mechanisms that underlie plasticity during the critical period.

Electrophysiological studies of the rodent visual system show that cortical neurons develop well-defined functional properties relating to eye preference and orientation selectivity, although

\footnotetext{
Received May 14, 2001; revised Nov. 28, 2001; accepted Dec. 7, 2001.

This work was supported by grants from the National Eye Institute, the Ellison Medical Foundation, and the Alfred P. Sloan Foundation (E.N.). We are grateful to members of the Nedivi laboratory and to Drs. Hollis T. Cline, Martha ConstantinePaton, and Rachel Wong for critical review of this manuscript. We thank Matthew Colonnese for help with Elvax surgeries.

Correspondence should be addressed to Elly Nedivi, Massachusetts Institute of Technology, E18-670, 50 Ames Street, Cambridge, MA 02139. E-mail: nedivi@mit.edu.

Copyright (ㄷ) 2002 Society for Neuroscience $\quad 0270-6474 / 02 / 221807-09 \$ 15.00 / 0$
}

stronger dependence on retinally driven action potentials. Dark rearing does not affect cpg 15 mRNA expression in the LGN and SC at any age, but it does significantly affect its expression in the visual cortex from the peak of the critical period and into adulthood. In dark-reared rats, the peak level of cpg15 expression in the visual cortex at P28 is lower than in controls. Rather than showing the normal decline with maturation, these levels are maintained in dark-reared animals. We suggest that the prolonged plasticity in the visual cortex that is seen in darkreared animals may result from failure to downregulate genes such as cpg15 that could promote structural remodeling and synaptic maturation.

Key words: dark rearing; visual system; cpg15; critical period; cortex; plasticity; TTX; monocular deprivation they are not anatomically segregated according to these properties as is seen in the visual cortex (VC) of cats and monkeys (Parnavelas et al., 1981; Maffei et al., 1992; Fagiolini et al., 1994; Gordon and Stryker, 1996; Antonini et al., 1999). In a manner similar to that in cats and monkeys, cells in the primary visual cortex of rodents are capable of undergoing shifts in eye preference during a critical period in development (Drager, 1978; Fagiolini et al., 1994; Gordon and Stryker, 1996; Guire et al., 1999). Anatomical studies show that in mice, monocular deprivation (MD) during the critical period affects arbor growth of thalamocortical afferents (Antonini et al., 1999). These studies, in combination with new technologies for manipulating mouse genetics, argue that the rodent visual projection can be a valuable experimental system for examining the cellular and molecular mechanisms of developmental plasticity.

Candidate plasticity gene 15 (cpg15) was isolated in a screen for seizure-induced genes in the dentate gyrus of the rat hippocampus (Nedivi et al., 1993). cpg15 expression is regulated by light in the visual cortex of adult rats (Nedivi et al., 1996); its localization and regulation in the developing visual system of cats are consistent with a role in activity-dependent plasticity (Corriveau et al., 1999). cpg15 overexpression in the developing Xenopus optic tectum induces exuberant growth of dendritic arbors in tectal cells (Nedivi et al., 1998); this is accompanied by enhanced growth of retinal axon arbors and retinotectal synapse formation (Cantallops et al., 2000). The effects of cpg15 on these different aspects of circuit formation are all non-cell autonomous, consistent with its glycosylphosphatidylinositol cell-surface attachment (Nedivi et al., 1998; Cantallops et al., 2000).

The potential role of cpg15 in dendritic and axonal arbor restructuring and synaptic maturation led us to study the effect of 
dark rearing on its expression and regulation. We monitored cpg15 mRNA levels in the visual structures of the rat before, during, and after the cortical critical period for the development of eye-specific preference. We compared the effects of dark rearing with those of retinal action potential blockade and MD. Our results suggest a mechanism whereby the visual cortex of DR animals maintains a capacity for delayed plasticity through elevated levels of genes such as cpg15 that can enhance local circuit remodeling and new synaptic stabilization. These results may provide a clue to the intriguing discrepancy seen in DR animals between the capacity for change as measured by anatomical methods and that measured by electrophysiological methods.

\section{MATERIALS AND METHODS}

Animal manipulations and tissue isolation. All animal work was approved by the Massachusetts Institute of Technology Committee on Animal Care; it conforms to National Institutes of Health guidelines for the use and care of vertebrate animals. Wistar-Kyoto rats (Taconic, Germantown, NY) were housed either in a room with a $12 \mathrm{hr}$ light/dark cycle or in a room sealed from visible light. A $15 \mathrm{~W}$ safelight shielded by a number 2 Kodak dark-red filter (Eastman Kodak, Rochester, NY) was used for the daily care and maintenance of animals housed in the darkroom $(\sim 30$ $\mathrm{min} / \mathrm{d}$ ). At various points during the experiment, Polaroid (Cambridge, MA) photographic paper placed in the darkroom was monitored for exposure. In cats, intermittent exposure to a safelight can be sufficient to prevent the apparent extension of the critical period by dark rearing. To test whether exposure to a safelight affected the dark rearing of rats, a group of animals handled with an infrared viewing system $(950 \mathrm{~nm})$ was raised in the dark to $3,4,5$, and 8 weeks of age (each group, $n=3$ ). The levels of $\operatorname{cpg} 15$ expression in the rats handled with intermittent exposure to a safelight were not significantly different from those handled under infrared conditions; both were significantly different from their agematched controls at 4 and 8 weeks (data not shown). The difference in sensitivity to exposure to a safelight may reflect the relatively poor vision of albino rats compared with cats.

Rats were reared under normal conditions (12 hr light/dark cycle), dark-reared from birth, or dark-adapted at different developmental time points. DR and age-matched control animals were killed postnatally at progressive times, starting at postnatal day 10 (P10) and at 1 week intervals from P14 (the approximate day of eye opening) to 8 weeks (P10, each group, $n=2$; P14, P21, 5 weeks, 6 weeks, each group, $n=4 ; 4$ weeks, DR, $n=4$; controls, $n=3$; 8 weeks DR, $n=3$; controls, $n=5$ ). A group of DR 8-week-old rats was exposed to light for 6, 12, or $24 \mathrm{hr}$ or for 7 or $14 \mathrm{~d}$ ( $n=2-3$ for each time point). Dark-adapted adult rats were placed in the dark for 2 weeks and killed in the dark, with a parallel set killed after re-exposure to $24 \mathrm{hr}$ of light (each group, $n=3$ ). Rats that were raised as dark-adapted until adulthood were raised normally to 2, 3, 4 , or 5 weeks; placed in the dark until 8 weeks; and killed in the dark or after re-exposure to light for $24 \mathrm{hr}(n=3$ per group per time point). Two additional groups of rats were allowed a 1 week window of visual experience [between weeks 3 and 4 (Lt3-4) or between weeks 4 and 5 (Lt4-5)] during dark rearing. At 8 weeks, these animals were killed in the dark (each group, $n=3$ ) or after $24 \mathrm{hr}$ of exposure to light (Lt3-4, $n=3 ;$ Lt4 $-5, n=2)$.

Monocular blockades of retinal action potentials were done essentially as described previously (Prusky and Ramoa, 1999). A strip of Elvax containing either tetrodotoxin (TTX) or citrate buffer was surgically implanted into the vitreous of the left eye of each animal for $3 \mathrm{~d}$ of sustained release, starting at P11, P18, P25, P32, P39, and P53 (TTX, $n=$ 3 ; citrate, $n=2$ ). Briefly, $200 \mathrm{mg}$ of washed Elvax beads (DuPont NEN, Wilmington, DE) were dissolved in methylene chloride. The dissolved Elvax was mixed with $20 \mu \mathrm{l}$ of $1 \%$ Fast Green in dimethylsulfoxide and either $20 \mu \mathrm{l}$ of $0.3 \mathrm{M}$ TTX in citrate buffer (Calbiochem, La Jolla, CA) or $20 \mu \mathrm{l}$ of $18.6 \mathrm{~mm}$ citrate buffer (Sigma, St. Louis, MO). The methylene chloride was slowly evaporated over the course of $1 \mathrm{~d}$ at $-70^{\circ} \mathrm{C}$ and for $5 \mathrm{~d}$ subsequently at $-20^{\circ} \mathrm{C}$. The Elvax was then sectioned into $180-\mu \mathrm{m}-$ thick disks by cryostat and stored at $-80^{\circ} \mathrm{C}$. Before surgeries, the Elvax was washed in $70 \%$ ethanol for $30 \mathrm{~min}$ and twice in sterile PBS for 30 min. For younger animals, the Elvax was cut into $\sim 2 \times 0.75 \mathrm{~mm}$ strips. For older animals, the Elvax strips were $\sim 4 \times 1 \mathrm{~mm}$. The strips were carefully inserted into the vitreous after making a small incision at the edge of the sclera. One eye in each animal was treated with either TTX or citrate Elvax; the other eye remained intact. Anesthesia was maintained by halothane $/ \mathrm{O}_{2}$ via mask. After Elvax implantation, the rats recovered from anesthesia under observation. The effectiveness of activity blockade was monitored daily by assaying for consensual pupil response to bright white illumination under light halothane $/ \mathrm{O}_{2}$ anesthesia.

MD by eyelid suture was initiated at P18, P25, P32, P39, and P53 (for each time point, $n=3)$. Under ketamine/xylazine $(80 / 10 \mathrm{mg} / \mathrm{kg})$ anesthesia, the area surrounding the left eye was cleaned with povidoneiodine and isopropyl alcohol. The lid margins were trimmed and the eye was flushed with sterile PBS. Two to three horizontal mattress sutures using 6.0 Ethilon (Johnson \& Johnson, Somerville, NJ) closed the length of the apposed lids. Ophthalmic ointment (Fougera, Melville, NY) was applied and the animals were monitored for recovery. For the P14 time point $(n=3)$, rather than suture the unopened eye, tissue adhesive (Vetbond; 3M, St. Paul, MN) was applied at P11 to prevent possible eye opening. After $3 \mathrm{~d}$ of TTX blockade or MD, animals were decapitated by guillotine; the brains were removed immediately and trimmed and positioned for coronal sectioning before being frozen on powdered dry ice and stored at $-80^{\circ} \mathrm{C}$.

In situ hybridization. Coronal sections $(10 \mu \mathrm{m})$ through the anterior visual cortex were sectioned by cryostat, thaw-mounted on Superfrost/ plus microscope slides (VWR Scientific, West Chester, PA), dried, fixed in $4 \%$ paraformaldehyde, washed in PBS, dehydrated in ethanol, airdried, and stored desiccated at $-80^{\circ} \mathrm{C}$. Before hybridization, slides were pretreated (at room temperature, unless otherwise stated) with $0.2 \mathrm{M}$ $\mathrm{HCl}(20 \mathrm{~min})$, double distilled water (DDW) $(5 \mathrm{~min}), 2 \times \mathrm{SSC}(30 \mathrm{~min}$ at $70^{\circ} \mathrm{C}$ ), and DDW (5 min). The next prehybridization treatments, from pronase (type XIV) (Sigma) to air-drying slides for $1 \mathrm{hr}$, were conducted as described previously (Hogan et al., 1994). RNA probes were synthesized with an RNA transcription kit (Stratagene, La Jolla, CA) and ${ }^{35} \mathrm{~S}-U T P(800 \mathrm{Ci} / \mathrm{mmol}$; Amersham Biosciences, Piscataway, NJ), using linearized cpg15 cDNA as a template. Hybridizations were done as described previously (Nedivi et al., 1996). Posthybridization wash conditions were as follows: $3 \mathrm{hr}$ at $50^{\circ} \mathrm{C}$ in $50 \%$ formamide and $1 \times$ salt solution (Hogan et al., 1994) with $10 \mathrm{mM}$ DTT; $15 \mathrm{~min}$ at $37^{\circ} \mathrm{C}$ in TNE (10 mm Tris, pH 7.5, $0.5 \mathrm{M} \mathrm{NaCl}, 1 \mathrm{~mm}$ EDTA); $30 \mathrm{~min}$ at $37^{\circ} \mathrm{C}$ in TNE containing RNase A $\left(20 \mu \mathrm{g} / \mathrm{ml}\right.$; Sigma); $30 \mathrm{~min}$ at $37^{\circ} \mathrm{C}$ in TNE; and finally overnight at $50^{\circ} \mathrm{C}$ in $50 \%$ formamide and $1 \times$ salt solution. Slides were dehydrated with $0.3 \mathrm{M} \mathrm{NH}_{4} \mathrm{Ac}$ in ethanol, air-dried, and processed for autoradiography as described previously (Hogan et al., 1994), using autoradiographic emulsion type NTB-2 (Eastman Kodak) diluted 1:1 with $2 \%$ glycerol, and exposed for $3-5 \mathrm{~d}$ at $4^{\circ} \mathrm{C}$.

Quantitative data analysis. Dark-field images of two to four sections from each brain were imported into Adobe Photoshop 5.0 (Adobe Systems, San Jose, CA) with a Diagnostic Instruments (Sterling Heights, MI) Spot2 digital camera mounted on a Nikon (Tokyo, Japan) Eclipse E600 using a $1 \times / 0.04$ Plan ultrawide objective. Images were saved as gray-scale TIFFs and imported into NIH Image (version 1.62). In addition to the VC and the superior colliculus (SC), each section contained the medial geniculate body (MGB) of the thalamus, an auditory sensory area (Fig. 1). Areas were defined by Nissl staining of alternate sections. Mean pixel density measurements were taken from four areas on each section: the VC, the SC, the background, and the MGB. Pixel density was measured on a $0-255$ scale, on which 255 is white. The background served as a zero-labeling negative control, whereas the MGB served as a positive control with a high level of labeling that is unaffected by visual manipulations. VC and SC measurements were normalized on a scale of $0-1$ interpolating between the background (0) and MGB (1) values. The background mean pixel density was first subtracted from the mean pixel densities in the $\mathrm{VC}$, the SC, and the MGB, yielding the net mean pixel densities for each area. The net mean pixel density in the VC or SC was then divided by the net mean pixel density from the MGB in the same section. Statistical significance was determined by unpaired Student's $t$ test. To confirm that measurements were unbiased, image files from four groups ( 4 week and 8 week controls, and 4 week and 8 week DR) were coded, mixed, and remeasured by technicians who were unaware of the experimental treatment. All data sets were not significantly different between blind and nonblind measurements. 


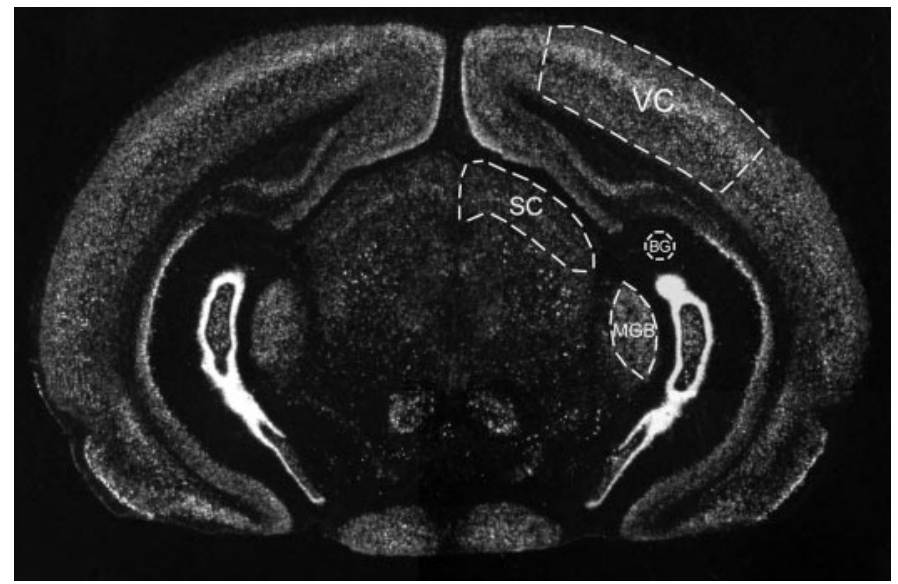

Figure 1. In situ hybridization on a coronal section through rat visual cortex using a $\operatorname{cpg} 15$ probe. The regions relevant for quantification are outlined. Mean pixel densities were measured in four areas on each section: the $\mathrm{VC}$, the $\mathrm{SC}$, the $\mathrm{MGB}$, and an area of the section with zero labeling for background $(B G)$. The background is used as the zero point, whereas the MGB served as a positive control with a high level of labeling that is unaffected by visual manipulations (see Materials and Methods).

\section{RESULTS}

\section{Developmental profile of $\operatorname{cpg} 15$ expression}

To investigate a role for cpg15 in the activity-dependent phase of the development of the rat visual system, in situ hybridizations with a cpg15 probe were conducted on sections through visual structures starting at P10. The lateral geniculate nucleus (LGN), $\mathrm{SC}$, and $\mathrm{VC}$ were monitored before and after eye opening, throughout the critical period for development of eye-specific preference in the binocular zone of primary VC, and in the adult. At birth and up to P10, expression of cpg15 is undetectable in the neocortex (Nedivi et al., 1996). At P10, 4 d before eye opening, low levels of cpg15 mRNA were visible at the apex of the cortical hemispheres (Fig. 2). General onset of $\operatorname{cpg} 15$ expression in the VC occurred coincident with eye opening at 2 weeks after birth, with the highest expression in layers $2 / 3$, 4, and 6. Subsequently, cpg15 mRNA expression levels gradually increased, peaking at P28, the height of the physiologically characterized critical period for the development of eye-specific preference in the VC. cpg15 mRNA levels then declined to a lower basal adult level. These results show that cpg15 levels in the rat VC correspond well with the electrophysiologically mapped critical period.

\section{Effect of dark rearing on cpg15 expression}

To test whether regulation of $\operatorname{cpg} 15$ expression was sensitive to visual input, rats were deprived of visual experience by dark rearing to various developmental ages. cpg15 mRNA levels were compared in DR animals and age-matched controls. Dark rearing did not alter the time of onset or the early increase in levels of cpg15 expression in the VC (Fig. 2). At P28, cpg15 mRNA levels in DR animals plateaued, but at a significantly lower level of expression than in age-matched controls (Figs. 2 and $3 a$ ). This level of expression persisted in older DR animals, so that after 8 weeks of dark rearing, cpg15 expression levels were significantly higher than in their age-matched controls, in which cpg15 expression normally declined after P28 (Figs. 2 and $3 a$ ). Differences in cpg15 mRNA levels between normal and DR animals were not seen in the LGN or SC (data not shown). The enhanced expres-
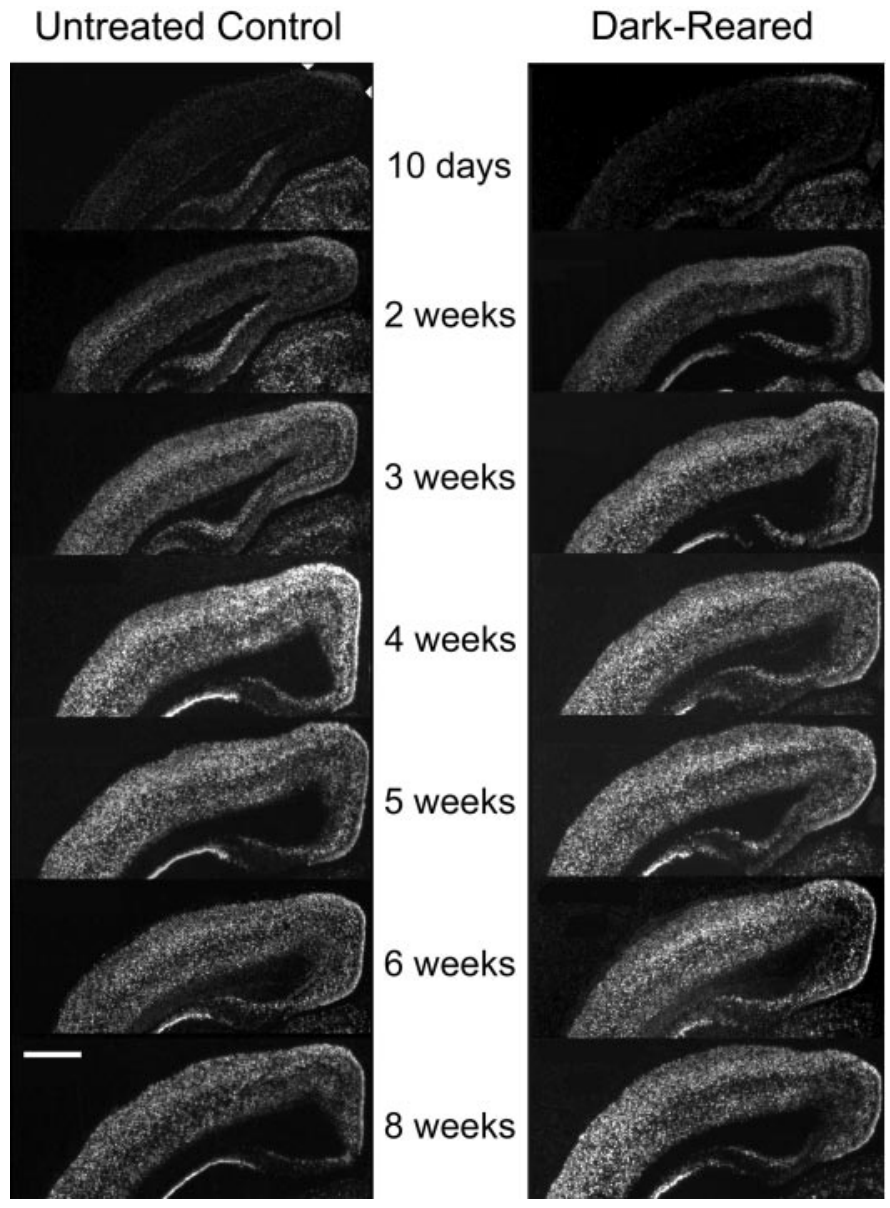

Figure 2. Developmental time course of $\operatorname{cpg} 15$ expression in the VC of normal and DR rats. Representative dark-field photomicrographs of in situ hybridizations for cpg15 mRNA are shown. Coronal sections through the VC of normal animals (left) and dark-reared animals (right) are shown at the designated postnatal ages. The onset of cpg15 expression in the neocortex for both normal and DR animals is coincident with eye opening at 2 weeks of age. Normally, cpg15 expression peaks at 4 weeks and then declines to a lower basal adult level. In DR animals, cpg15 levels remain elevated past 5 weeks. Arrowheads point to the earliest cpg15 expression at the medial apex of the neocortex. Some individual panels (untreated, 6 weeks; DR, 6 weeks; untreated, 4 weeks; DR, 4 weeks; DR, 3 weeks; and DR, 2 weeks) were normalized with respect to the MGB for assembly of the montage. Scale bar, $1 \mathrm{~mm}$.

sion levels of $\operatorname{cpg} 15$ in the VC, seen with dark rearing, may be a molecular indicator of the prolonged plasticity specific to this region.

Reportedly, a short exposure to light can trigger the end of the delayed plasticity seen in DR animals (Mower et al., 1983; Philpot et al., 2001). To test whether a late onset of visual experience affects cpg15 expression, animals were dark-reared to 8 weeks and then exposed to light for 6,12 , or $24 \mathrm{hr}$ or for 7 or $14 \mathrm{~d}$. In situ hybridizations show that with exposure to light of up to $12 \mathrm{hr}$, cpg15 expression remains significantly higher than normal (Fig. 4). After $24 \mathrm{hr}$ of exposure to light, DR animals showed cpg15 mRNA expression levels that were comparable with those found in normally raised adults (Fig. 4). These experiments indicate that brief visual experience in DR adults can reset cpg15 expression to normal levels. This downregulation of expression may be a molecular representation of the light-induced end of the delayed plasticity in DR animals. 
a

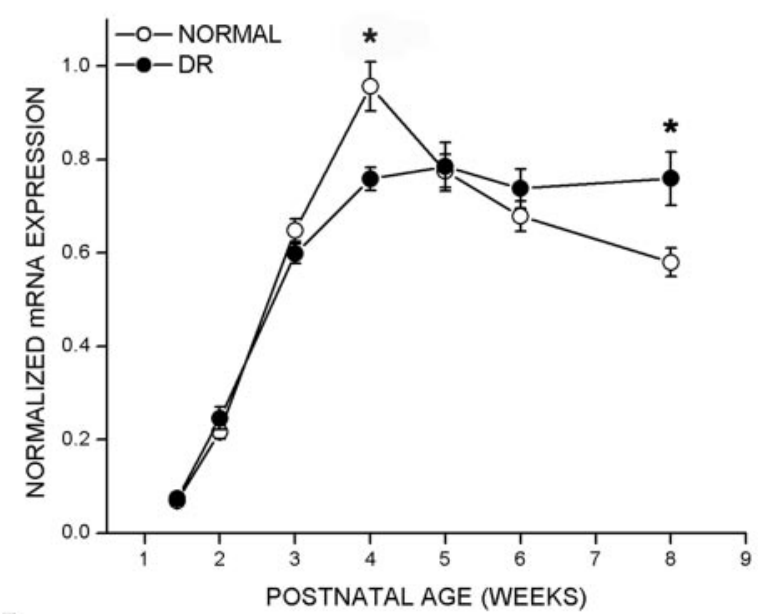

b

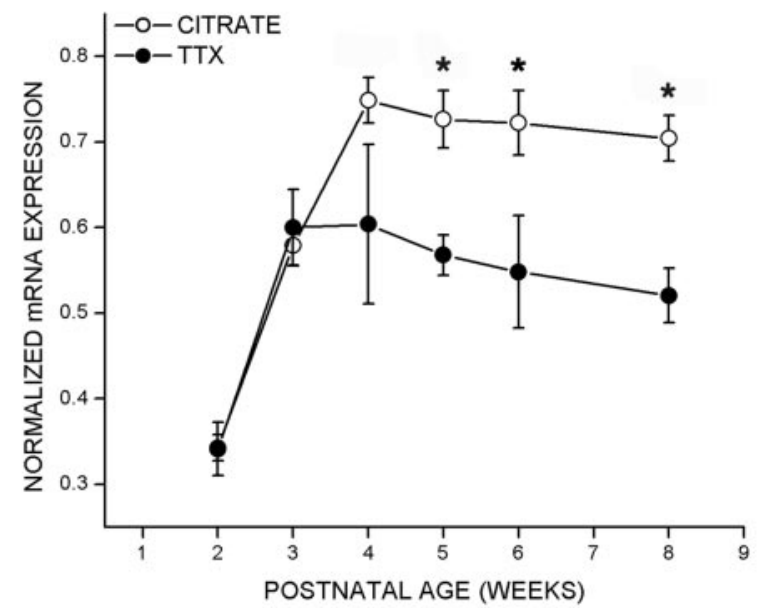

C

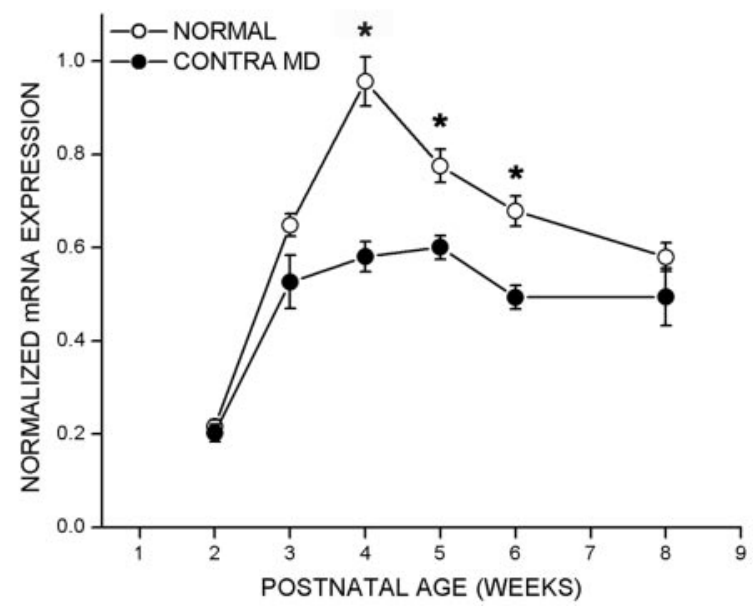

Figure 3. Quantification of cpg15 expression in the VC of control (normal) rats, DR rats, rats after blockade of retinal activity, or rats after MD. In all cases, after background subtraction, the net average pixel value in the VC was normalized by the net average pixel value of the MGB (see Materials and Methods for details). a, Effect of dark rearing on cpg15 expression in the VC. Data from four in situ experiments are shown,

\section{Effect of blockade of retinal action potentials on cpg15 expression}

To investigate whether cpg15 mRNA expression in the VC is dependent on retinally driven action potential activity, the $\mathrm{Na}^{+}$ channel blocker TTX was applied to the left retina of normally raised rats for $3 \mathrm{~d}$ periods, starting at different developmental times. Age-matched control animals were treated with citrate buffer applied to the left retina. cpg15 mRNA levels in the VC contralateral to the TTX-treated eye were compared with those in the VC contralateral to the citrate-treated eye in control animals. There was no change in the onset of cpg15 expression in response to monocular retinal activity blockade, and there was no significant influence on cortical levels of cpg15 mRNA during early postnatal development (Fig. 5). Mean levels of cpg15 expression at 2, 3, and 4 weeks after birth were not significantly different between the VC contralateral to the blocked eye and the VC contralateral to the citrate-treated controls (Fig. 3b). In contrast, after the peak of the critical period and into adulthood, monocular retinal action potential blockade decreased cpg15 expression in the $\mathrm{VC}$ contralateral to the blocked eye (Figs. $3 b$ and 5).

Monocular TTX blockade also decreased cpg15 expression in the contralateral LGN and SC starting 3 weeks after birth. For both the LGN and superficial layers of the SC contralateral to the treated eye, the effect of TTX blockade became more pronounced with age (LGN not shown; for SC, see Figs. 5 and 6). These experiments indicate that in all of the visual structures tested, the developmental regulation of $\operatorname{cpg} 15$ expression is divided into two phases. Early cpg 15 expression is independent of retinally driven action potentials. During the critical period for development of eye-specific preference in the VC, an activity-dependent component of cpg15 expression emerges, and the effect of retinal action potential blockade becomes progressively more pronounced with age.

\section{Effect of monocular deprivation on cpg15 expression}

It has been shown that total blockade of retinal activity produces a smaller shift in ocular dominance plasticity than that produced by MD (Rittenhouse et al., 1999). To investigate whether the effect of monocular activity blockade on cpg15 expression is less extreme than that of MD, we monocularly deprived normally raised rats for $3 \mathrm{~d}$ periods, starting at different developmental times. cpg15 levels in the $\mathrm{VC}$ and the SC contralateral to the

of which Figure 2 is representative. Solid circles, DR animals; open circles, untreated control animals. Error bars indicate the SEM. Points marked with asterisks are significantly different between normal and DR animals (at 4 weeks, $p=0.001$; at 8 weeks, $p=0.016$; unpaired Student's $t$ test). $b$, Effect on cpg15 expression in the VC of monocular TTX blockade for $3 \mathrm{~d}$ initiated at different developmental times. Data from three in situ experiments are shown, of which Figure 5 is representative. Solid circles, VC contralateral to TTX blockade; open circles, $\mathrm{VC}$ of control animals implanted with a citrate control in the contralateral eye. Error bars indicate the SEM. Points marked with asterisks are significantly different between the VC of control and TTX-treated rats (at 5 weeks, $p=0.0012$; at 6 weeks, $p=0.032$; at 8 weeks, $p=0.0008$; unpaired Student's $t$ test). $c$, Effect on cpg15 expression in the VC of $\mathrm{MD}$ for $3 \mathrm{~d}$ by eyelid suture initiated at different developmental time points. Solid circles, VC contralateral to the sutured eye; open circles, $\mathrm{VC}$ of untreated control animals. Error bars indicate the SEM. Points marked with asterisks are significantly different between the VC of control and MD rats (at 4 weeks, $p=0.003$; at 5 weeks, $p=0.006$; at 6 weeks, $p=0.007$; unpaired Student's $t$ test). 


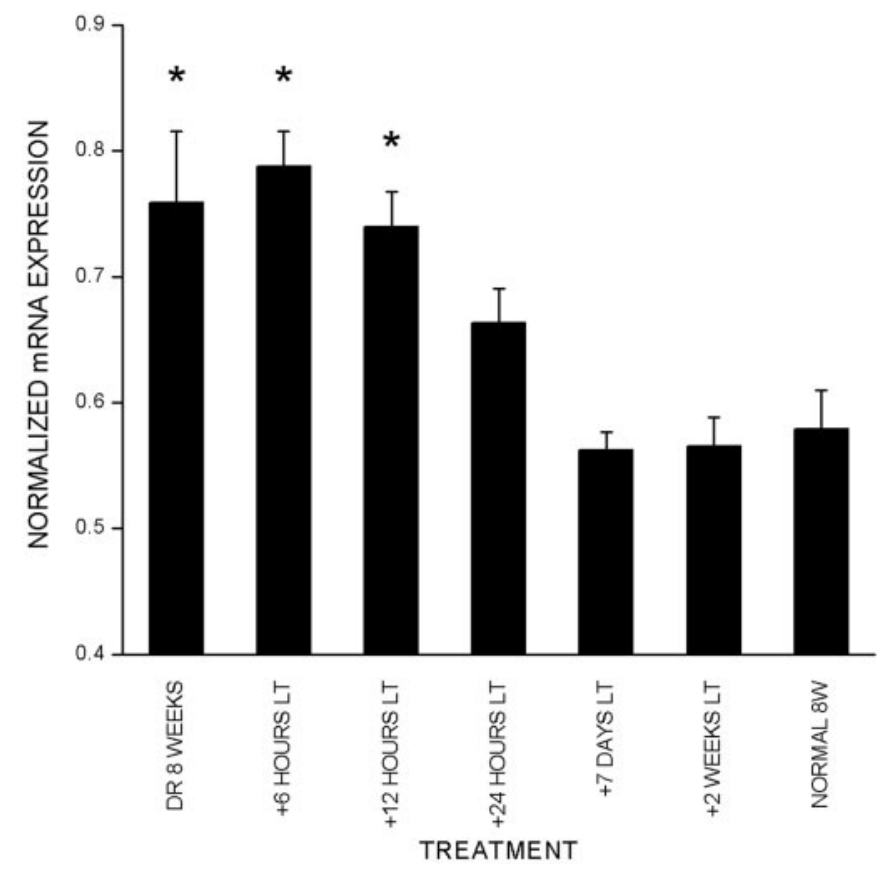

Figure 4. Light triggers downregulation of $\operatorname{cpg} 15$ expression in DR rats. Quantification of $\operatorname{cpg} 15$ expression in the VC of animals DR to 8 weeks and subsequently exposed to light $(L T)$ for designated times. Data from two in situ experiments are shown. In each section, the net average pixel value in the VC is normalized by the net average pixel value of the MGB. Abnormally high levels of $\operatorname{cpg} 15$ expression in animals dark-reared to 8 weeks decline to normal adult levels within $24 \mathrm{hr}$ of exposure to light. Error bars indicate the SEM. Asterisks mark significant differences compared with normal animals at 8 weeks (DR, 8 weeks, $p=0.016$; DR, 8 weeks plus $6 \mathrm{hr}$ of light, $p=0.009$; DR, 8 weeks plus $12 \mathrm{hr}$ of light, $p=$ 0.0086; unpaired Student's $t$ test).

sutured eye were compared with the VC and the SC in normal animals. As in both DR and TTX-treated animals, there was no change in the onset of cpg15 expression in the VC of MD rats (Fig. 3c). During the next 4 weeks of development, cpg15 expression in the VC contralateral to the MD eye was significantly less than in normal animals. Unlike animals whose retinally driven action potentials are blocked by TTX, at the peak of the critical period (P28) MD animals exhibit levels of cpg15 expression that are significantly less than those of controls. With maturation, the effect of MD becomes less significant, and at 8 weeks after birth, cpg15 expression in the deprived VC is no longer different from controls. This trend is opposite to the effect of TTX, which becomes more significant as the animals mature. As in DR animals and in contrast to animals with retinal activity blockade, monocular deprivation did not affect cpg15 levels in the contralateral SC.

\section{Effect of early visual experience on adult cpg15 expression}

Previous studies suggest that early periods of visual experience are sufficient to trigger closure of the critical period despite later visual deprivation (Mower et al., 1983; Mower and Christen, 1985). To determine the window of early visual experience required for the development of normal adult regulation of cpg15 expression in the VC, animals were raised in a normal 12 hr light/dark cycle to 2, 3, 4, or 5 weeks after birth and then transferred to a dark environment until adulthood at 8 weeks. Animals were killed without seeing any additional light or after

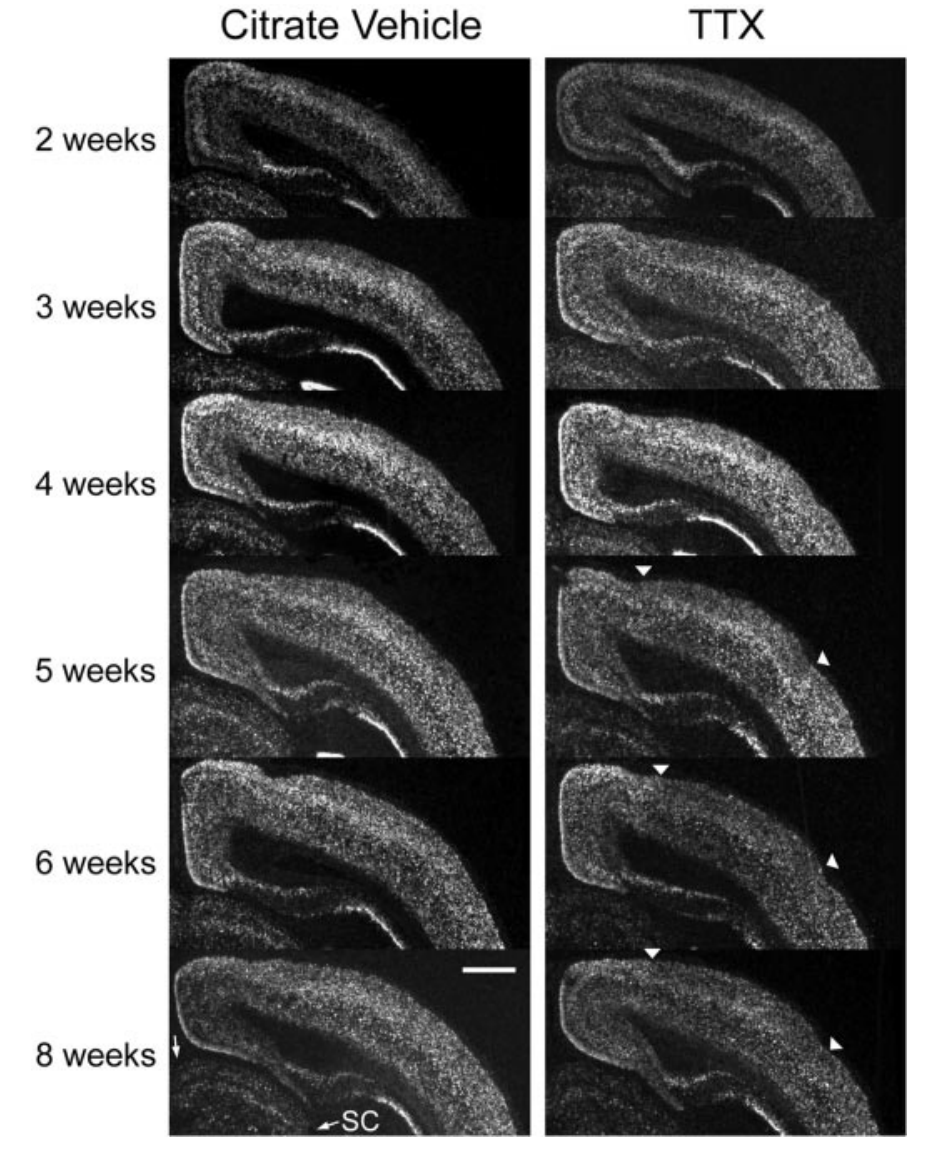

Figure 5. cpg15 expression in the $\mathrm{VC}$ after $3 \mathrm{~d}$ of monocular TTX blockade initiated at different developmental times. Dark-field photomicrographs of in situ hybridizations for cpg 15 mRNA in coronal sections through the $\mathrm{VC}$ contralateral to the treated eye of either control animals (left) implanted with the citrate vehicle or TTX-treated animals (right), at the designated ages. Starting at 5 weeks after birth, retinal TTX blockade decreases levels of cpg15 expression in the VC (see cortical area between arrowheads). Starting at 4 weeks after birth, retinal TTX blockade causes a downregulation of $\operatorname{cpg} 15$ expression in the SC ( $S C$ indicated by $t w o$ arrows). Some individual panels (citrate, 4 weeks; TTX, 4 weeks; TTX, 3 weeks) in this figure were normalized with respect to the MGB for assembly of the montage. Scale bar, $1 \mathrm{~mm}$.

$24 \mathrm{hr}$ of re-exposure to light, to assess their ability to regulate cpg15 expression. In normally raised adult rats, cpg15 mRNA expression in the $\mathrm{VC}$ is downregulated after a 2 week period in the dark. After re-exposure to light for $24 \mathrm{hr}$, such darkadapted rats will upregulate cpg15 levels (Nedivi et al., 1996) (Figs. $7 h$ and $8 h$ ). In contrast, rats raised in darkness to 8 weeks show abnormally high levels of cpg15 expression in the VC; after exposure to $24 \mathrm{hr}$ of light, the levels of expression are decreased (Figs. 4, 7a, and 8a). These two extremes were compared with animals exposed to visual experience at restricted windows during development. Animals exposed to visual experience for 2 or 3 weeks before being placed in the dark until they had reached adulthood displayed a regulation of cpg15 expression that was essentially the same as that seen in DR animals (Figs. $7 b, c$, and $8 b, c$ ). Animals reared with normal visual experience for the first 4 or 5 weeks after birth and then placed in darkness until 8 weeks of age regulate $\operatorname{cpg} 15$ expression in the VC similarly to normal animals (Figs. 7d,e and $8 d, e)$. These results suggest that 1 week of visual experience at the outset of the critical period is sufficient to confer 


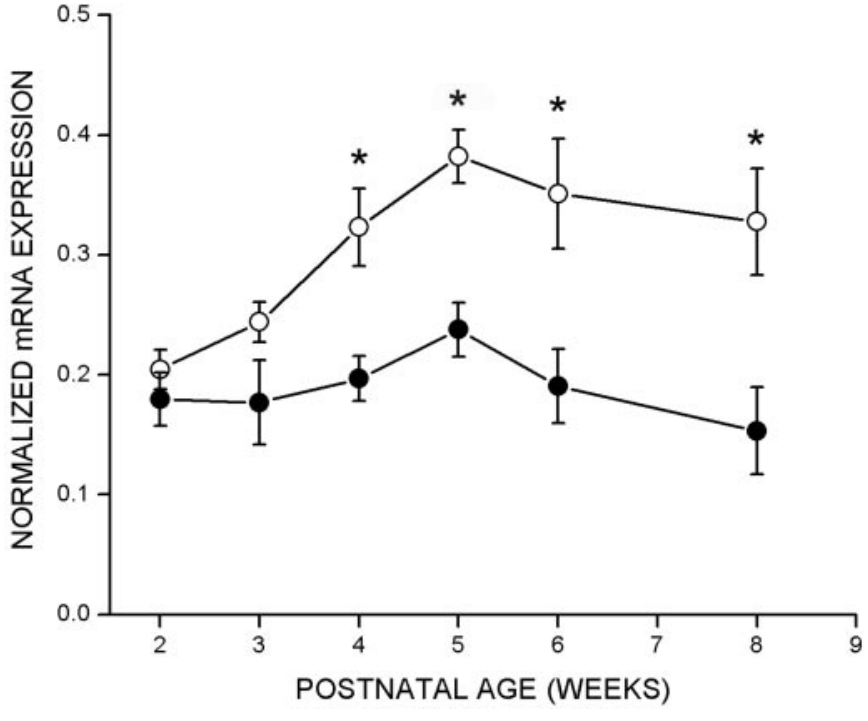

Figure 6. Quantification of cpg15 expression in the SC after $3 \mathrm{~d}$ of monocular TTX blockade initiated at different developmental times. Data from three in situ experiments are shown, of which Figure 5 is representative. After background subtraction, the net average pixel value in superficial layers of the SC was normalized by the net average pixel value of the MGB. cpg15 expression in the SC is depressed by retinal TTX blockade soon after eye opening. Solid circles, SC contralateral to TTX blockade; open circles, SC contralateral to citrate control. Error bars indicate SEM. Points marked with asterisks are significantly different between the SC of control and TTX-treated rats (at 4 weeks, $p=0.026$; at 5 weeks, $p=0.0003$; at 6 weeks, $p=0.027$; at 8 weeks, $p=0.011$; unpaired Student's $t$ test).

normal patterns of adult cpg15 regulation. To test this prediction, animals were raised to 8 weeks in darkness except for 1 week of light either between weeks 3 and 4 or between weeks 4 and 5. cpg 15 expression in the $\mathrm{VC}$ was assayed at 8 weeks. cpg15 mRNA levels in animals allowed just 1 week of visual experience during the critical period are strikingly similar to those seen in normally raised animals and are dramatically induced on re-exposure to light for $24 \mathrm{hr}$ (Fig. 8f,g). Our results show that a restricted 1 week window of visual experience during the critical period is sufficient to determine adult patterns of cpg15 regulation in the VC.

\section{DISCUSSION}

The experiments described here address the temporal and spatial expression patterns of cpg15 in the developing visual system of the normal rat and in response to visual manipulations. cpg15 expression was monitored in relation to the critical period for development of eye-specific preference in the VC. We compared expression during normal development with that seen in response to dark rearing, monocular retinal action potential blockade, or MD. The regulation of cpg15 expression patterns suggests that it may serve as a molecular indicator of the potential for visual system plasticity.

\section{Activity-independent and activity-dependent phases of cpg15 expression}

We found that in the visual system of rats, the onset of cpg15 mRNA expression in the VC occurs 2 weeks after birth. cpg15 expression levels gradually rise and peak 2 weeks later, in the midst of the electrophysiologically mapped cortical critical period for shifts in eye preference. After its peak expression, cpg15
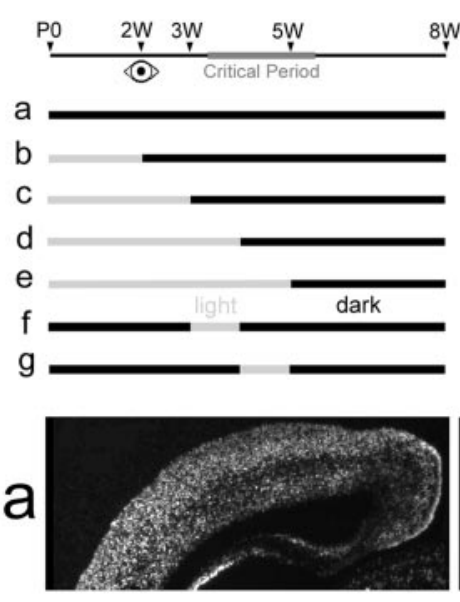

$+24 \mathrm{hr}$ light
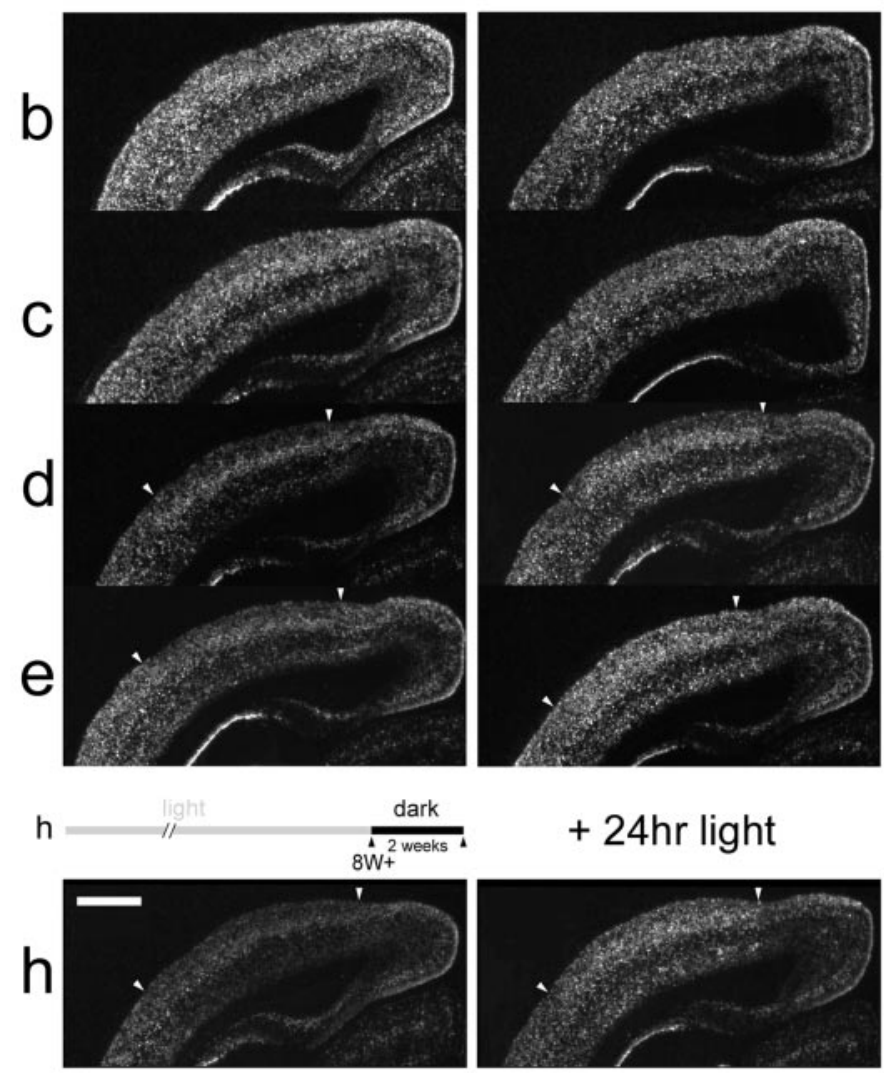

Figure 7. Early visual experience confers normal adult patterns of cpg15 regulation. Representative dark-field photomicrographs of in situ hybridizations for cpg15 mRNA on coronal sections through the VC are shown. Animals were raised to different ages with normal visual experience and then transferred into a dark environment until 8 weeks of age (left), followed by a $24 \mathrm{hr}$ re-exposure to light (right). Animals raised normally to 4 or 5 weeks of age $(d, e)$ and then dark-reared to 8 weeks show the normal decline in cpg15 levels with maturation as well as the normal adult regulation $(h)$ of cpg15 expression by light (arrowheads delineate the VC). Similar to DR animals from birth $(a)$, animals raised normally to 2 and 3 weeks of age $(b, c)$ and then dark-reared to 8 weeks fail to downregulate cpg15 as adults and do not reinduce cpg15 in response to light. Panels were not normalized for montage assembly. Scale bar, $1 \mathrm{~mm}$.

mRNA levels in the VC decline to a lower basal level in adults, concomitant with critical period closure at $\sim 6$ weeks of age (Fagiolini et al., 1994; Gordon and Stryker, 1996). Although the onset of cpg15 expression is coincident with eye opening, it is 


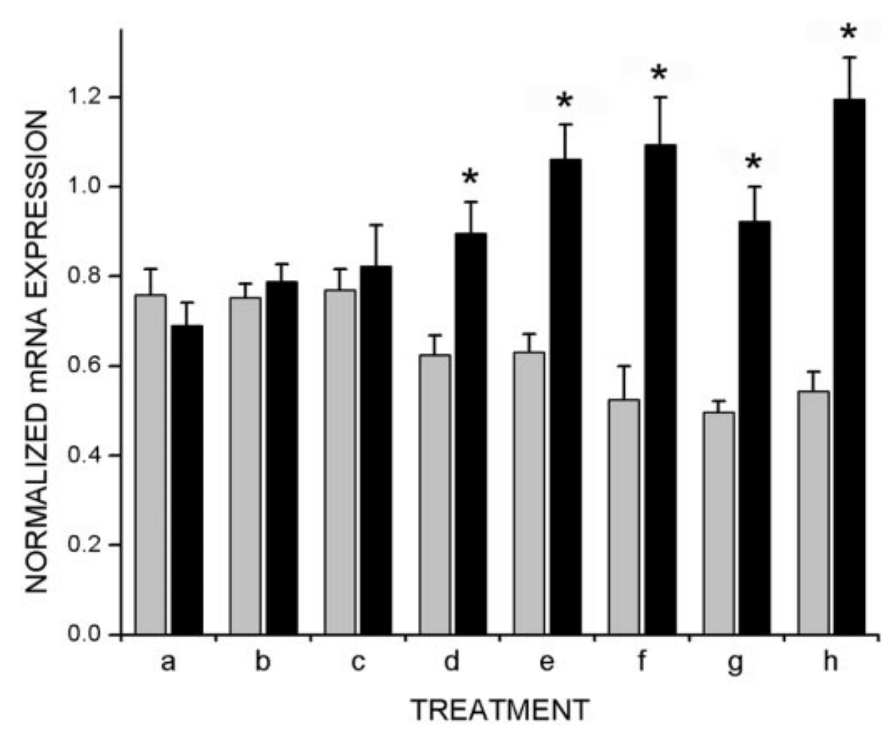

Figure 8. One week of visual experience during the critical period is sufficient to confer the normal adult patterns of $\operatorname{cpg} 15$ regulation. Quantification of $\operatorname{cpg} 15$ expression in the VC of rats with early visual experience. Measurements and calculations were done as described in Figure 3. The treatment legend is identical to Figure 7. A 1 week window of light during the critical period, either between weeks 3 and $4(f)$ or weeks 4 and $5(\mathrm{~g})$, confers normal adult patterns of cpg15 regulation. Gray bars, cpg15 expression levels without re-exposure to light; black bars, levels after $24 \mathrm{hr}$ of light exposure. Error bars indicate the SEM. Points marked with asterisks are significantly different between animals killed in the dark or after $24 \mathrm{hr}$ of light exposure $(e, h, p<0.0001 ; f, p=0.0049 ; g, p=0.0022$; $d, p=0.009$; unpaired Student's $t$ test $)$.

unaffected by dark rearing, blockade of retinally driven action potentials, or MD. This is consistent with previous studies of cpg15 expression in the visual system of cats, which demonstrated that early cpg 15 expression in the $\mathrm{VC}$ is activity independent (Corriveau et al., 1999). Therefore, the initial timing of cpg15 expression is likely to be controlled by a developmentally regulated activity-independent mechanism. This fits with electrophysiological and optical imaging studies that demonstrate that the basic structure of cortical maps is innate and develops in the absence of visual experience (Crair et al., 1998).

These studies also show that experience is necessary at a later stage of development for the refinement of ocular selectivity and to maintain responsiveness (Crair et al., 1998). We find that after the peak of the critical period, as the levels of cpg15 in the VC begin to decline, a component of cpg15 expression that is dependent on retinally driven action potentials becomes evident and is progressively more pronounced with age. Activity-dependent regulation of cpg15 expression in the SC and the LGN can be detected 1 week earlier than in the VC, perhaps because of the earlier maturation of these visual structures. Our results indicate that the regulation of $\operatorname{cpg} 15$ expression is biphasic in all visual structures. Early cpg 15 expression is independent of retinally driven action potentials. With maturation during the critical period, an activity-dependent component of cpg15 expression emerges, and the effect of the blockade of retinally driven action potentials becomes progressively more pronounced with age. Activity-dependent regulation of cpg15 arises relatively late in development and may represent an adult feature of visual system plasticity.

\section{Effect of visual experience on cpg15 expression}

The aspects of $\operatorname{cpg} 15$ regulation that we found to be dependent on normal visual experience were its peak levels in the VC at P28 and its subsequent downregulation after the end of the critical period. Although cpg15 levels in the VC of DR rats are indistinguishable from those in control rats within the first 3 weeks after birth, the expression at P28 is lower than in controls. Rats DR beyond P28 maintain the same peak level of cpg15 expression through adulthood, levels that are significantly higher than in their control counterparts. There is a crossover of the cpg15 expression profiles in normal and DR rats, such that at the peak of the critical period expression is higher in normal animals, whereas after the critical period cpg15 is higher in DR animals. This crossover can also be seen in the developmental profiles of susceptibility to MD in normal and DR cats (Mower, 1991). In rodents, there have been no studies that examine the effects of dark rearing on susceptibility to MD during the height of the critical period. Studies at later ages show that similar to cats, DR rats also retain a prolonged capability to respond to $\mathrm{MD}$, even at P90 (Guire et al., 1999).

With respect to dark rearing, we show here two additional cases in which cpg15 regulation closely parallels plasticity as measured by susceptibility to MD. Electrophysiological studies have demonstrated that a short exposure to light can trigger the end of the delayed plasticity that results from dark rearing (Mower et al., 1983; Philpot et al., 2001). Similarly, early visual experience in DR kittens attenuates the effects of later dark rearing so that there is no delayed plasticity (Cynader, 1983). These results show that the effect of dark rearing can be negated by a short period of light exposure in DR adults, or with sufficient early visual experience. We find that a $24 \mathrm{hr}$ exposure to light returns cpg15 levels in DR rats to those found in normally raised adults, and that 1 week of visual experience during the critical period is sufficient to confer normal adult patterns of $\operatorname{cpg} 15$ regulation.

Our results show that even 1 week of visual experience during the critical period is sufficient for establishing adult patterns of cpg15 regulation. This suggests that visual experience is not persistently required during development for normal functional maturation of the visual system to occur. Rather, exposure to patterned vision for at least 1 week during the critical period can irreversibly trigger the molecular machinery that is required for maturation; this will likely result in normal adult responses to visual manipulations. Because cpg15 expression is abnormally high in the adult in the absence of visual experience, the molecular trigger for maturation may involve a general downregulation of plasticity genes such as cpg15.

\section{Differences in the effect of DR on cpg15 from effects of activity blockade and MD}

The effect on cpg15 expression of binocular elimination of patterned vision during the critical period is profoundly different from the effect of retinal action potential blockade or MD. Dark rearing causes a prolonged upregulation of cpg15 expression starting at the peak of the critical period and continuing into adulthood, whereas retinal action potential blockade at the same developmental times causes a decrease in cpg15 expression. The effect of dark rearing is exclusive to the VC, whereas blockade of retinally driven action potentials affects cpg15 expression in the LGN and the SC as well as in the VC. This result corresponds well with the observation that the delayed plasticity seen in DR 
cats is not manifested in the LGN (Mower et al., 1985; Mower and Christen, 1985).

During the critical period, downregulation of cpg15 expression in the VC by MD is more severe than that caused by retinal activity blockade. This is consistent with electrophysiological studies that show that MD during the critical period produces a greater shift of ocular dominance in the VC than monocular blockade of retinal activity (Rittenhouse et al., 1999). A possible explanation is that during MD the residual activity from the retina actively depresses the efficacy of synaptic connections driven by the deprived eye (Bear and Rittenhouse, 1999; Rittenhouse et al., 1999). Despite the fact that an MD eye is generating some activity as opposed to the total loss of activity caused by the blockade of retinally driven action potentials, during the critical period cpg15 levels are lower in the $\mathrm{VC}$ of MD animals than in animals after retinal TTX blockade. This could reflect the depression of cortical synaptic activity driven by the deprived eye.

Taken together, all of this indicates that regulation of cpg15 expression does not correspond directly to levels of activity. Rather, it seems to reflect a propensity for functional plasticity.

\section{Effect of dark rearing on expression of visually responsive genes}

It has been proposed that mechanisms that underlie adult plasticity during learning and memory, or long-term potentiation and long-term depression, also play a key role in developmental plasticity (Kandel and O'Dell, 1992; Goodman and Shatz, 1993; Constantine-Paton and Cline, 1998; Nedivi, 1999). For this reason, many genes isolated or characterized on the basis of their response to activity in the adult have been investigated in the context of developmental plasticity in the visual system. These include both regulatory genes that encode transcription factors as well as effector genes that can directly affect neuronal morphology and function (for review, see $\mathrm{Ne}$ divi, 1999). Multiple genes show a transcriptional response to dark rearing, although the type of response varies. Whereas growth-associated protein 43 (GAP43), calcium calmodulindependent kinase II (CaMKII), and glutamic acid decarboxylase $(G A D)$ all show the same increase in expression shown by cpg15 in response to dark rearing past the critical period (Neve and Bear, 1989), junB, zif/268, and BDNF show the opposite response and are downregulated (Rosen et al., 1992; Lein and Shatz, 2000). In contrast to the light-independent onset of cpg15 expression, dark rearing prevents the normal onset and transcriptional increase of Homer, zif/268, and BDNF in the VC (Worley et al., 1990; Brakeman et al., 1997; Capsoni et al., 1999; Lein and Shatz, 2000). Subsequent exposure to light causes their rapid induction (Worley et al., 1990; Brakeman et al., 1997; Capsoni et al., 1999; Lein and Shatz, 2000). The transcriptional regulation of this latter group provides an accurate "molecular readout" of activity, whereas regulation of cpg15 together with $G A P 43, C a M K I I$, and $G A D$ corresponds more closely with the capacity for plasticity.

\section{Summary}

During the development of the Xenopus visual system, cpg15 concurrently regulates multiple aspects of retinotectal circuit formation (Cantallops et al., 2000). It promotes tectal cell dendritic arbor growth, stabilizes retinal axon arbors, and promotes matu- ration of retinotectal synapses (Nedivi et al., 1998; Cantallops et al., 2000). Our finding that DR rats fail to downregulate cpg15 raises the possibility that perhaps the residual plasticity measured electrophysiologically in these animals reflects an extended capacity for local synaptic remodeling. The prolonged plasticity seen in DR animals may result from failure to downregulate genes such as cpg15, which could promote structural remodeling and synaptic maturation.

\section{REFERENCES}

Antonini A, Fagiolini M, Stryker MP (1999) Anatomical correlates of functional plasticity in mouse visual cortex. J Neurosci 19:4388-4406.

Bear MF, Rittenhouse CD (1999) Molecular basis for induction of ocular dominance plasticity. J Neurobiol 41:83-91.

Brakeman PR, Lanahan AA, O’Brien R, Roche K, Barnes CA, Huganir RL, Worley PF (1997) Homer: a protein that selectively binds metabotropic glutamate receptors. Nature 386:284-288.

Cantallops I, Haas K, Cline HT (2000) Postsynaptic CPG15 promotes synaptic maturation and presynaptic axon arbor elaboration in vivo. Nat Neurosci 3:1004-1011.

Capsoni S, Tongiorgi E, Cattaneo A, Domenici L (1999) Dark rearing blocks the developmental down-regulation of brain-derived neurotrophic factor messenger RNA expression in layers IV and V of the rat visual cortex. Neuroscience 88:393-403.

Constantine-Paton M, Cline HT (1998) LTP and activity-dependent synaptogenesis: the more alike they are, the more different they become. Curr Opin Neurobiol 8:139-148.

Corriveau R, Shatz CJ, Nedivi E (1999) Dynamic regulation of cpg15 during activity-dependent development in the mammalian visual system. J Neurosci 19:7999-8008.

Crair MC, Gillespie DC, Stryker MP (1998) The role of visual experience in the development of columns in cat visual cortex. Science 279:566-570.

Cynader M (1983) Prolonged sensitivity to monocular deprivation in dark-reared cats: effects of age and visual exposure. Brain Res Dev Brain Res 8:155-164.

Cynader M, Mitchell DE (1980) Prolonged sensitivity to monocular deprivation in dark-reared cats. J Neurophysiol 43:1026-1040.

Drager UC (1978) Observations on monocular deprivation in mice. J Neurophysiol 41:28-42.

Fagiolini M, Pizzorusso T, Berardi N, Domenici L, Maffei L (1994) Functional postnatal development of the rat primary visual cortex and the role of visual experience: dark rearing and monocular deprivation. Vision Res 34:709-720.

Goodman CS, Shatz CJ (1993) Developmental mechanisms that generate precise patterns of neuronal connectivity. Cell 10:77-98.

Gordon JA, Stryker MP (1996) Experience-dependent plasticity of binocular responses in the primary visual cortex of the mouse. J Neurosci 16:3274-3286.

Guire ES, Lickey ME, Gordon B (1999) Critical period for the monocular deprivation effect in rats: assessment with sweep visually evoked potentials. J Neurophysiol 81:121-128.

Hogan B, Beddington R, Constantini F, Lacy E (1994) Manipulating the mouse embryo. In: A laboratory manual, Ed 2, pp 344-351. New York: Cold Spring Harbor Laboratory.

Hubel DH, Wiesel TN (1970) The period of susceptibility to the physiological effects of unilateral eye closure in kittens. J Physiol (Lond) 206:419-436.

Hubel DH, Wiesel TN, LeVay S (1977) Plasticity of ocular dominance columns in monkey striate cortex. Philos Trans R Soc Lond B Biol Sci 278:377-409.

Kandel ER, O’Dell TJ (1992) Are adult learning mechanisms also used for development? Science 258:243-245.

Lein ES, Shatz CJ (2000) Rapid regulation of brain-derived neurotrophic factor mRNA within eye-specific circuits during ocular dominance column formation. J Neurosci 20:1470-1483.

Maffei L, Berardi N, Domenici L, Parisi V, Pizzorusso T (1992) Nerve growth factor (NGF) prevents the shift in ocular dominance distribution of visual cortical neurons in monocularly deprived rats. J Neurosci 12:4651-4662.

Mower GD (1991) The effect of dark rearing on the time course of the critical period in cat visual cortex. Brain Res Dev Brain Res 58:151-158.

Mower GD, Christen WG (1985) Role of visual experience in activating critical period in cat visual cortex. J Neurophysiol 53:572-589.

Mower GD, Berry D, Burchfiel JL, Duffy FH (1981) Comparison of the effects of dark rearing and binocular suture on development and plasticity of cat visual cortex. Brain Res 220:255-267.

Mower GD, Christen WG, Caplan CJ (1983) Very brief visual experience eliminates plasticity in the cat visual cortex. Science 221:178-180. 
Mower GD, Caplan CJ, Christen WG, Duffy FH (1985) Dark rearing prolongs physiological but not anatomical plasticity of the cat visual cortex. J Comp Neurol 235:448-466.

Nedivi E (1999) Molecular analysis of developmental plasticity in neocortex. J Neurobiol 41:135-147.

Nedivi E, Hevroni D, Naot D, Israeli D, Citri Y (1993) Numerous candidate plasticity-related genes revealed by differential cDNA cloning. Nature 363:718-722.

Nedivi E, Fieldust S, Theill L, Hevroni D (1996) A set of genes expressed in response to light in the adult cerebral cortex and regulated during development. Proc Natl Acad Sci USA 93:2048-2053.

Nedivi E, Wu GY, Cline H (1998) Promotion of dendritic growth by CPG15, an activity-induced signaling molecule. Science 281:1863-1866

Neve RL, Bear MF (1989) Visual experience regulates gene expression in the developing striate cortex. Proc Natl Acad Sci USA 86:4781-4784.
Parnavelas JG, Burne RA, Lin CS (1981) Receptive field properties of neurons in the visual cortex of the rat. Neurosci Lett 27:291-296.

Philpot BD, Sekhar AK, Shouval HZ, Bear MF (2001) Visual experience and deprivation bidirectionally modify the composition and function of NMDA receptors in visual cortex. Neuron 29:157-169.

Prusky GT, Ramoa AS (1999) Novel method of chronically blocking retinal activity. J Neurosci Methods 87:105-110.

Rittenhouse CD, Shouval HZ, Paradiso MA, Bear MF (1999) Monocular deprivation induces homosynaptic long-term depression in visual cortex. Nature 397:347-350.

Rosen KM, McCormack MA, Villa-Komaroff L, Mower GD (1992) Brief visual experience induces immediate early gene expression in the cat visual cortex. Proc Natl Acad Sci USA 89:5437-5441.

Worley PF, Cole AJ, Murphy TH, Christy BA, Nakabeppu Y, Baraban JM (1990) Synaptic regulation of immediate-early genes in brain. Cold Spring Harb Symp Quant Biol 55:213-222. 